\title{
On Water Density Fluctuations with Helices of Hydrogen Bonds
}

\author{
Alexander Shimkevich and Inessa Shimkevich \\ Division of Advanced Nuclear Energy Systems, National Research Center "Kurchatov Institute", 1 Kurchatov Square, \\ Moscow 123182, Russia \\ Correspondence should be addressed to Alexander Shimkevich, shall@dhtp.kiae.ru
}

Received 29 March 2011; Revised 2 June 2011; Accepted 10 June 2011

Academic Editor: Ivan Smalyukh

Copyright (c) 2011 A. Shimkevich and I. Shimkevich. This is an open access article distributed under the Creative Commons Attribution License, which permits unrestricted use, distribution, and reproduction in any medium, provided the original work is properly cited.

\begin{abstract}
An adaptive model is developed here for the liquid water density fluctuations as momentary dense clusters with helices of hydrogen bonds and nondense tetrahedral clusters of ice. This model can be useful for explanation of liquid water structural anomalies including the high quantity of hydrogen bonds with quasitetrahedral orientation in the nonordered liquid water. The topology of such clusters is essentially differed from the one of the crystalline ice. From this and only this point of view, the liquid water can be considered as a two-structural fluid by dynamic forming the two topological kinds of clusters as a consequence of condensedmatter density fluctuations. Another feature of the dense-water-part clusters is helical ordering of protons which can realize coherent vibrations. A spectral series of such vibrations is determined as a function of the number of molecules into the helical cluster.
\end{abstract}

\section{Introduction}

Although the liquid water is all around us, we still do not understand how it behaves on a microscopic level. Its dynamic hydrogen bonds (HB) structure has been the subject of intense debate for decades. Ice with well-established HB structure forms a tight tetrahedral lattice of water molecules each binding to four others. The prevailing model of the liquid water holds that the molecules loosen their grip but remain generally arranged in the same tetrahedral groups [1].

However, the majority of water molecules in the liquid are found in higher density regions [2]. The structure and states of the molecules in these regions are found through $\mathrm{X}$ ray emission spectroscopy (XES) and X-ray Raman spectroscopy (XRS, BL6-2), techniques which allows to analyze electronic states of molecules. The researchers have found that the molecules in the high density regions form an asymmetrical disordered structure where some islands of tetrahedral order are floated. This new research is requiring some sort of secondary structure. The greater density of liquid water implies that the molecules are more closely packed than the simple tetrahedrons seen in the ice $[3,4]$.
The conclusion that a dominant fraction of molecules in the liquid water is very asymmetrically hydrogen bonded with only two well-defined $\mathrm{H}$ bonds (one donating and one accepting) is in a strong contrast to the accepted picture of four quasitetrahedral $\mathrm{H}$ bonds.

Very recently, also X-ray emission spectroscopy (XES) is applied at high resolution to the liquid water [5], arriving at the similar conclusions as $[1,2]$. They interpret the observed splitting of the lone-pair state into two sharp features as evidence of a bimodal distribution of local structures in the liquid: either strongly tetrahedral or highly disordered, which interconvert discontinuously with temperature. From smallangle X-ray scattering (SAXS) studies, they have found evidence for density nonhomogeneity on a length scale of $1-2 \mathrm{~nm}$ indicating that the two components are spatially separated on the time scale of the experiment $[3,4]$.

The controversy about the microstructure of liquid water pits a new model involving water molecules in a relatively stable "rings-and-chains" structure against the standard model that considers water molecules in the distorted tetrahedral coordination. An analysis [6] and molecular dynamics (MD) simulations $[7,8]$-both classical and $a b$ initio-almost uniformly support the standard model. 
At the same time, it is known that a topological densityfluctuations structure of liquids is ramified clusters of almost regular Delaunay simplexes (tetrahedrons) built on the fours of densely packed particles and connected in pairs by faces in tetrahedral Bernal chains $[9,10]$. The Voronoi-Delaunay method is used for analyzing atomic configurations in disordered systems. The review of publications on this subject is presented in the monograph [11].

For identifying the dense-part structure of liquids, they have selected those Delaunay simplexes which have a tetrahedral factor more than or equal to the some given one, $K_{T}<1$ (convention criterion). Then, it is possible to build atomic ensembles in the MD model of liquids and investigate their dense-part structure by choosing assorted simplexes of the regular subdivision in snapshots of the MD cell [10-13].

Opposite, a topological criterion offered in [13-16] for finding simplexes of the atomic-ensemble dense part exactly (rigorous criterion) defines dense-part simplexes on the overall length of their edges in maximum of number of tetrahedral clusters in the MD cell. Then, one can get a statistics of these clusters for different liquids.

These data may be useful for creating an analytical model of the cluster statistics for calculating the configuration entropy of any condensed matter and estimating its thermodynamic properties using only a pair potential of particles [17] and for analyzing the microstructure features of water [18].

\section{Same Structural Features of the Liquid Water}

Precise experimental techniques for determining the local structure of the liquid water are lacking since each water molecule undergoes a quick rearrangement (during femtoseconds). The need for better understanding the liquid water at the microscopic level has forced developing computational methods that describe the individual and cooperative dynamics and structure of water molecules. Many investigations are carried out using these techniques. They show that locally HB-ordered clusters of water molecules are formed and broken uninterruptedly [19].

MD studying density fluctuations of the liquid water have shown [18] that the low-density tetrahedral-ordered ice regions are divided by higher density tetrahedral clusters with ramified structure (see Figure 1). It is obvious that these dense-part clusters of water density fluctuations are more complicated due to hydrogen bonds.

\section{Helical Model for the Dense Part of Liquid Water}

One can expect that water molecules have enough time for rebuilding hydrogen bonds in the dense part of water density fluctuations due to fast librations and rotations of them. From this, we will build a model for tetrahedral clusters of the dense part of liquid water provided that each water molecule in such cluster is hydrogen bonded with three molecules of this cluster and all angles between these bonds are equal to each other.

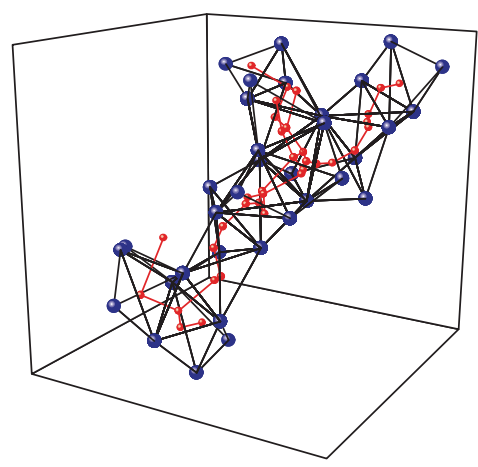

Figure 1: The dense-part cluster in MD model of water at $300 \mathrm{~K}$ and its frame (broken red line); blue points are the molecules and red points are centers of the cluster tetrahedrons.

It turned out that such cluster represents "nanotube" of water molecules and geometric parameters of this tube are defined strictly. For illustration, Figure 2 shows an angular shift, $\gamma$, for coordinates $\left(x^{2}, y^{2}\right)$ of second-class particles relative to coordinates $\left(x^{1}, y^{1}\right)$ of first-class particles which are defined as

$$
\begin{gathered}
\vec{r}_{k}^{1}:\left\{\begin{array}{l}
x_{k}^{1}=a \rho \cos \left[\left(\frac{4 \pi}{5}-\beta\right) k\right] \\
y_{k}^{1}=a \rho \sin \left[\left(\frac{4 \pi}{5}-\beta\right) k\right] \\
z_{k}^{1}=a \Delta k
\end{array}\right. \\
\vec{r}_{k}^{2}:\left\{\begin{array}{l}
x_{k}^{2}=a \rho \cos \left[\left(\frac{4 \pi}{5}-\beta\right) k-\gamma\right] \\
y_{k}^{2}=a \rho \sin \left[\left(\frac{4 \pi}{5}-\beta\right) k-\gamma\right] \\
z_{k}^{2}=a \Delta k+a \sqrt{1-4 \rho^{2} \sin ^{2}\left(\frac{\gamma}{2}\right)} .
\end{array}\right.
\end{gathered}
$$

We obtain a helix of hydrogen bonds with the radius, $\rho$, in units of $\mathrm{HB}$ distance, $a$; $\triangle$ is the shift value (on the helix axis " $z$ ") of the following first-class particle in the same units; $\beta$ is the angular correction of first-class particle shift in the plane $(x, y) ; \gamma$ is the angular shift of the second-class particle projection on the plane $(x, y)$ relative to the first-class (see Figure 2) with the same index, $k$.

These parameters are defined under the assumption of helix edges equality as the distance between oxygen atoms of nearest water molecules: $a=\left|\vec{r}_{k}^{1}-\vec{r}_{k-2}^{2}\right|=\left|\vec{r}_{k+1}^{1}-\vec{r}_{k-2}^{2}\right|$ and angles between them:

$$
\begin{gathered}
\cos \alpha_{1}=\left(\frac{1}{a^{2}}\right)\left(\vec{r}_{k}^{1}-\vec{r}_{k-2}^{2}\right)\left(\vec{r}_{k+1}^{1}-\vec{r}_{k-2}^{2}\right), \\
\cos \alpha_{2}=\left(\frac{1}{a^{2}}\right)\left(\vec{r}_{k}^{2}-\vec{r}_{k}^{1}\right)\left(\vec{r}_{k-2}^{2}-\vec{r}_{k}^{1}\right), \\
\cos \alpha_{3}=\left(\frac{1}{a^{2}}\right)\left(\vec{r}_{k+1}^{2}-\vec{r}_{k+1}^{1}\right)\left(\vec{r}_{k-2}^{2}-\vec{r}_{k+1}^{1}\right) .
\end{gathered}
$$




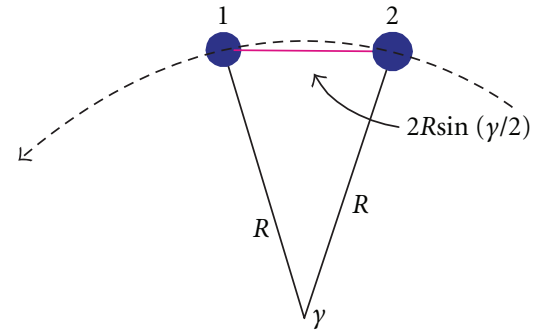

FIGURE 2: The diagram of angular shift, $\gamma$, for coordinates $\left(x^{2}, y^{2}\right)$ of second-class particles relative to coordinates $\left(x^{1}, y^{1}\right)$ of first-class particles.

From the equality of angles, $\alpha_{1}, \alpha_{2}$, and $\alpha_{3}$, and noting $\alpha \equiv \pi / 5+\beta$, one can obtain a system of four independent equations for defining the parameters: $\alpha, \gamma, \Delta$ и

$$
\begin{aligned}
0= & \Delta^{2}-\Delta \sqrt{1-4 \rho^{2} \sin ^{2}\left(\frac{\gamma}{2}\right)} \\
& +\rho^{2} \sin \alpha \sin (\alpha-\gamma), \\
0= & 9 \Delta^{2}-6 \Delta \sqrt{1-4 \rho^{2} \sin ^{2}\left(\frac{\gamma}{2}\right)} \\
& +\rho^{2}[2 \cos \gamma+2 \cos (3 \alpha-\gamma)], \\
0= & 6 \Delta^{2}-3 \Delta \sqrt{1-4 \rho^{2} \sin ^{2}\left(\frac{\gamma}{2}\right)} \\
& +\rho^{2}[-\cos \alpha-\cos 2 \alpha+\cos \gamma+\cos (3 \alpha-\gamma)], \\
0= & \Delta \sqrt{1-4 \rho^{2} \sin ^{2}\left(\frac{\gamma}{2}\right)} \\
& +\rho^{2}[\cos 2 \alpha+\cos 3 \alpha-\cos \gamma+2 \sin \alpha \sin (\alpha-\gamma) \\
& \quad-\cos (3 \alpha-\gamma)] .
\end{aligned}
$$

Solutions of this task for $\gamma$ and $\rho$ as functions of $\alpha$ and $\Delta$ are $\operatorname{tg}[(\alpha-\gamma) / 2]=(\cos \alpha+\cos 2 \alpha) /(\sin 2 \alpha+2 \sin \alpha)$ and $\rho^{2}=$ $(3 / 2) \Delta^{2} /(\cos \alpha+\cos 2 \alpha)$. Then, for $\alpha$, we have $0=12 \cos 3 \alpha+$ $16 \cos 2 \alpha-4 \cos \alpha-8$, which has a single real solution, $\cos \alpha=$ $2 / 3$. From here, we obtain $\beta=12.189685^{\circ} \approx 4 \pi / 59, \cos \gamma=$ $58 / 63, \Delta=7 / \sqrt{190}$, and $\rho=13 / \sqrt{190}$.

Using these parameters, it is easy to find the value of $\cos \alpha_{i}=-11 / 38$ which gives $\alpha_{i}=106.83^{\circ}$.

Now, one can estimate a packing factor of water molecules into the helix provided that all particles are inside the cylinder in radius of $\rho+0.5$, that is, when particles forming the helix belong to it entirely. Then, we have the bottom estimation of this factor as $\eta_{\text {helix }} \geq(2 k+4) /$ $k \Delta \pi(\rho+0.5)^{2}{ }_{k \rightarrow \infty}=2 / \pi \Delta(\rho+0.5)^{2}=0.70$.

In using the cubic model of ice shown in Figure 3, it is easy to estimate its packing factor for water molecules.

The elementary ice cell contains 7.5 molecules of water. The edge length of such cell is equal to $4 / \sqrt{3}$ per the unit of

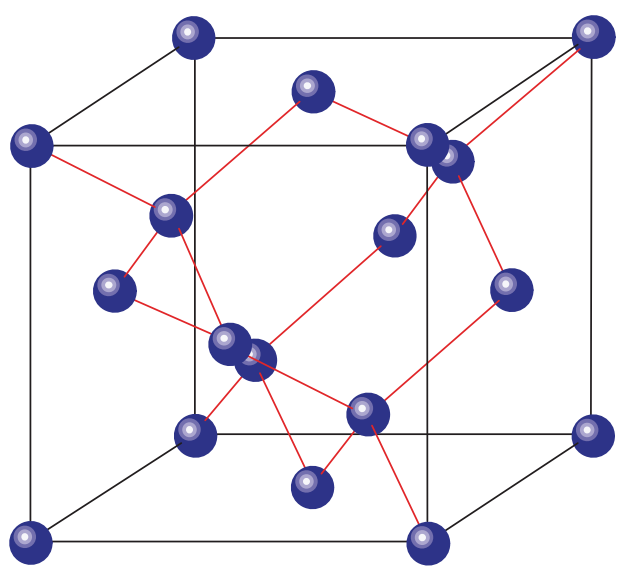

Figure 3: The cubic model of ice.
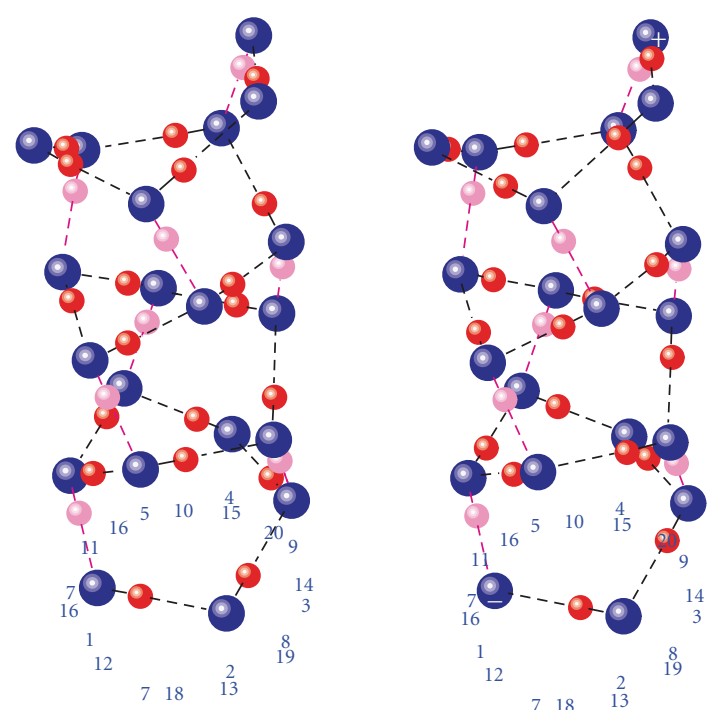

FIGURE 4: The dense part of water density fluctuations with the coherent exchange of protons (red balls) between oxygen atoms (blue balls) along the helical hydrogen bonds (black lines) and HB bridges (pink balls); the projections of oxygen atoms in the $(x, y)$ plane are denoted by numbers.

intermolecular distance. From here, we will find $\eta_{\text {ice }}=$ $7.5(\sqrt{3} / 4)^{3}=0.61$.

One can estimate a volume portion $\Omega$ of helical clusters as the dense part of liquid water from $1.0=(0.92 /$ $0.61)[0.70 \Omega+0.61(1-\Omega)]$, taking into account that the density of ice is equal to $0.92 \mathrm{~g} / \mathrm{cm}^{3}$ and the water one is equal to $1.0 \mathrm{~g} / \mathrm{cm}^{3}$ at $4^{\circ} \mathrm{C}$. From here, we obtain an upper bound of this portion as helical clusters $\Omega \leq(0.08 /$ $0.92)(0.61 / 0.09)=0.59$.

These clusters having higher molecular density provide, in principle, four hydrogen bonds for each molecule of water: three of them are internal and one is external for connecting ice crystallites with less density. However, if protons are ordered in the helix (see Figure 4), there can arise resonant vibrations of proton current in a coin of the helical cluster. One can estimate frequencies of these vibrations. 


\section{Evaluating Resonance Frequencies of the Coherent Proton Vibrations}

One assumes that the motion of all protons in helical circuit (coin), as shown in Figure 4, is synchronous; that is, the protons vibrate coherently in the helical cluster of hydrogen bonds for the dense part of liquid water. This hypothesis is based on anomalous proton polarizability in hydrogenbonded chains due to a collective proton motion [20-22]. Another basis is electromagnetic inducing the collective proton motion [23].

For describing such coherent vibrations, we can use equation [24]:

$$
\frac{1}{c^{2}} L \frac{d^{2} q}{d t^{2}}+\frac{q}{\varepsilon C}=0
$$

modified for oscillatory tunneling of heavy quantum particles (protons) through the potential barrier [25] with a permeability, $D$ :

$$
\frac{m_{p}}{m_{e} c^{2}} L \frac{d^{2} q}{d t^{2}}+\frac{D q}{\varepsilon C}=0
$$

Here, $m_{p}$ and $m_{e}$ are, respectively, proton and electron masses $c$ is the light velocity $L$ is the self-induction of closed circuit $C$ is its electrical capacity and $\varepsilon$ is the dielectric water permeability. A solution of (5) presents electromagnetic waves with frequency of

$$
\nu=c \sqrt{m_{\mathrm{e}} D / \varepsilon m_{p} L C} / 2 \pi
$$

For defining its value, we shall estimate the parameters in (5). It is known that $m_{p} / m_{e} \sim 1836, \varepsilon \sim 80, c \sim 3 \cdot 10^{10} \mathrm{~cm} / \mathrm{s}$. From defining $L$ and $C$ in [24], one can present them for given task as

$$
\begin{gathered}
L=\pi \mathrm{a}^{2} \rho^{2} N^{2} / l, \\
C=r_{\mathrm{w}} l / 2\left(l-r_{\mathrm{w}}\right) .
\end{gathered}
$$

Here, $\mathrm{a}=2.82 \AA, r_{\mathrm{w}}=1.41 \AA$, is the average radius of water molecule, $l=n \mathrm{a} \Delta$, $n$ is the number of water molecules in helical cluster (see Figure 4 ), and $N$ is the number of coin coils which is approximately equal to $n / 6$. An expression of $D$ is obtained in [26] as

$$
D=\left[1+\frac{\operatorname{sh}^{2}\left(\Lambda k_{2}\right)\left(k_{1}^{2}+k_{2}^{2}\right)^{2}}{4 k_{1}^{2} k_{2}^{2}}\right]^{-1},
$$

where $k_{1}=\sqrt{2 m_{p} \mathrm{E}} / \hbar, k_{2}=\sqrt{2 m_{p}\left(\mathrm{U}_{o}-\mathrm{E}\right)} / \hbar, \Lambda$ is equal to the length difference of covalent and hydrogen bonds, that is, $\Lambda=0.84 \AA, E=k_{B} T=0.42 \cdot 10^{-20} \mathrm{~J}$, and $U_{o}=$ $1.66 \cdot 10^{-20} \mathrm{~J}$, as one fourth energy difference of covalent and hydrogen bonds, $k_{B}$ is Boltzmann constant, and $T$ is Kelvin temperature.

In substituting these values in formula (8), we shall obtain $D \sim 1.1 \cdot 10^{-4}$.
TABLE 1: The spectral absorption modes of the coherent proton vibrations as a function of the water-molecules number in the helix, $n$.

\begin{tabular}{lcrccc}
\hline$n$ & \multicolumn{2}{c}{$\begin{array}{c}\text { Spectral modes } \\
\mathrm{cm}^{-1}\end{array}$} & $n$ & \multicolumn{2}{c}{ Spectral modes } \\
$\mathrm{THz}$ & 273 & 20 & 4.8 & $\mathrm{~cm}^{-1}$ \\
\hline 6 & 8.2 & 220 & 25 & 4.3 & 160 \\
10 & 6.6 & 183 & 30 & 3.9 & 144 \\
15 & 5.5 & & & \\
\hline
\end{tabular}

Finally, from (6), we find a spectral series of coherent vibrations of protons in helical clusters of the dense part of liquid water:

$$
v \sim \frac{22 \sqrt{n-1}}{n}[\mathrm{THz}]
$$

for $n \geq 6$. Then, we will receive the spectral absorption modes of coherent vibrating protons (Table 1).

\section{Discussion of Results}

The topology of the dense liquid water part as helices of hydrogen bonds differs from the one for tetrahedral crystalline-ice clusters as a nondense part of liquid water. Therefore, one can tell about water as a two-structural liquid though this is a dynamic distinction having only statistical sense because such clusters arise and disappear as density fluctuations of the liquid water.

At the same time, possible coherent proton vibrations by external impact at their resonant frequencies can selectively amplify some modes and, thus, strengthen water microheterogeneity far from its equilibrium. In this connection, studying the electromagnetic absorption spectra in this range of frequencies can be interested both for revealing spectral lines (9) and for searching for a possible technique for controlling the microstructure of the liquid water.

Moreover, any additives can be concentrated in the expected ramified border between two dynamic structures of water solution that, in turn, can cause fluctuation-induced clustering and impurity [26].

Thus, the helices of hydrogen bonds in water density fluctuations and the high resonant mobility of proton in them as a catalytic stimulator for chemical reactions can create templates for building macromolecules and structural blocks of biosystems.

\section{Conclusions}

The model of hydrogen bond helix is built for the dense part of the liquid water density fluctuations. This model allows providing the high quantity of tetrahedral hydrogen bonds in the nonordered liquid matrix and explaining the density anomaly of liquid water.

The topology of such helical clusters is essentially differed from the one of the crystalline ice. From this and only this point of view, the liquid water can be considered as the twostructural liquid because the formation and decay of such 
clusters has dynamic character and is natural consequence of water density fluctuations.

The other interesting feature of these clusters is possible helical ordering protons in them which can have coherent vibrations. A spectral series of such resonances is defined.

\section{Acknowledgments}

The authors are pleased to acknowledge Dr. A. S. Kolokol for giving some data on molecular dynamic simulation of water structure and discussing this work which is supported by the Russian Foundation of Basic Research (RFBR) (Grant no. 1008-00217a).

\section{References}

[1] C. Huang, K. T. Wikfeldt, T. Tokushima et al., "The inhomogeneous structure of water at ambient conditions," Proceedings of the National Academy of Sciences of the United States of America, vol. 106, no. 36, pp. 15214-15218, 2009.

[2] P. Wernet, D. Nordlund, U. Bergmann et al., "The structure of the first coordination shell in liquid water," Science, vol. 304, no. 5673, pp. 995-999, 2004.

[3] E. Cartlidge, "The strangest liquid," New Scientist, February 2010.

[4] K. Tuttle, "Researchers rediscover the structure of water," SLAC Today, February 2010.

[5] T. Tokushima, Y. Harada, O. Takahashi et al., "High resolution $\mathrm{X}$-ray emission spectroscopy of liquid water: the observation of two structural motifs," Chemical Physics Letters, vol. 460, no. 4-6, pp. 387-400, 2008.

[6] J. D. Smith, C. D. Cappa, B. M. Messer, W. S. Drisdell, R. C. Cohen, and R. J. Saykally, "Probing the local structure of liquid water by X-ray absorption spectroscopy," Journal of Physical Chemistry B, vol. 110, no. 40, pp. 20038-20045, 2006.

[7] H. A. Stern and B. J. Berne, "Quantum effects in liquid water: path-integral simulations of a flexible and polarizable ab initio model," Journal of Chemical Physics, vol. 115, no. 16, pp. 76227628, 2001.

[8] M. J. McGrath, J. I. Siepmann, I.-F. W. Kuo et al., "Simulating fluid-phase equilibria of water from first principles," Journal of Physical Chemistry A, vol. 110, no. 2, pp. 640-646, 2006.

[9] J. D. Bernal, "The structure of liquids," Proceedings of the Royal Society of London. Series A, vol. 280, no. 1382, pp. 299-322, 1964.

[10] Y. Waseda, The structure of non-crystalline materials; liquids and amorphous solids, McGraw-Hill, New York, NY, USA, 1980.

[11] N. N. Medvedev, Voronoy-Delaunay method in research of structure of non-crystalline systems Novosibirsk, Publishing House of the Siberian Branch of the Russian Academy of Science, 2000.

[12] N. P. Smolin, B. R. Gelchinski, A. A. Mirzoev, and E. V. Dyuldina, "The analysis of the short-range-order atomic structure of liquid metals by the Voronoi polyhedron method and a check of the adequacy of the results gained by the RMC method," Journal of Non-Crystalline Solids, vol. 312-314, pp. 90-94, 2002.

[13] A. S. Kolokol et al., "MD-simulation of liquid metals in the substantiation of a concept for designing liquid metal coolants," Preprint IAE-6281/11. Moscow, Russia, 2003.
[14] A. S. Kolokol and A. L. Shimkevich, "Topological structure of liquid metals," Atomic Energy, vol. 98, no. 3, pp. 187-190, 2005.

[15] A. L. Shimkevich, A. S. Kolokol, and I. Yu. Shimkevich, "Twostructure model for simple metals," Journal of Non-Crystalline Solids, vol. 353, no. 32-40, pp. 3472-3474, 2007.

[16] A. S. Kolokol and A. L. Shimkevich, "Topological structure of density fluctuations in condensed matter," Journal of NonCrystalline Solids, vol. 356, no. 4-5, pp. 220-223, 2010.

[17] A. L. Shimkevich, "Tetrahedral-chain-cluster model for thermodynamic description of fluids," in Proceedings of the 16th International Conference on Nuclear Engineering (ICONE '08), Orlando, Fla, USA, 2008, Paper ICONE16-48566.

[18] P. N. Alekseev et al., "On basic principles for modifying water as a coolant of PWR," in Transactions of European Nuclear Conference, vol. 4, p. 9, 2010.

[19] R. Roy, W. A. Tiller, I. Bell, and M. R. Hoover, "The structure of liquid water; novel insights from materials research; potential relevance to homeopathy," Materials Research Innovations, vol. 9, no. 4, pp. 98-103, 2005.

[20] G. Zundel and K. Leberle, "Proton polarizability of poly(Ltyrosine)-hydrogen phosphate hydrogen bonds as a function of alkali cations," Biopolymers, vol. 23, no. 4, pp. 695-705, 1984.

[21] G. Zundel, "Hydrogen bonds with large proton polarizability and proton transfer processes in electrochemistry and biology," Advances in Chemical Physics, vol. 111, pp. 1-217, 2000.

[22] M. Shaikh, J. Mohanty, D. K. Maity, S. K. Nayak, and H. $\mathrm{Pal}$, "Collective proton motion in the intramolecular hydrogen bonding network and the consequent enhancement in the acidity of hydroxycalixarenes," Journal of Photochemistry and Photobiology A, vol. 195, no. 1, pp. 116-126, 2008.

[23] G. Careri, "Correlations \& conductivity: geometric aspects of physics chemistry and biology," in Proceedings of the NATO Advanced Study Institute Held at Cargese, Corsica, France, $\mathrm{H}$. Stanley and N. Ostrowsky, Eds., Kluwer Academic Publishers, Dordrecht, The Netherlands, 1990.

[24] L. D. Landau and E. M. Lifshits, Electric Dynamics of Condensed Matter, GosTechLit, Moscow, Russia, 1957.

[25] K. Hyeon-Deuk and K. Ando, "Quantum effects of hydrogen atoms on the dynamical rearrangement of hydrogen-bond networks in liquid water," Journal of Chemical Physics, vol. 132, no. 16, Article ID 164507, 2010.

[26] A. L. Shimkevich, The Composition Principles for Designing Nuclear-Reactor materials, N. N. Ponomarev-Stepnoi, Ed., IzdAt, Moscow, Russia, 2008. 

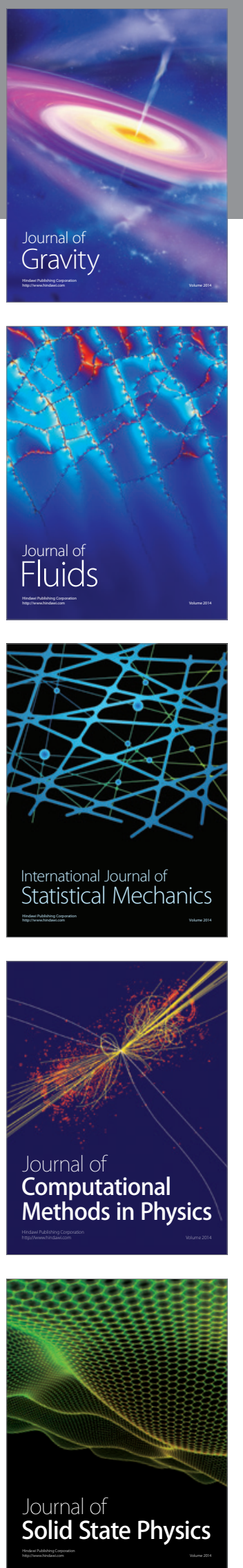

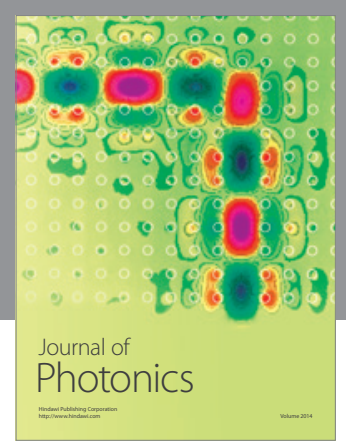

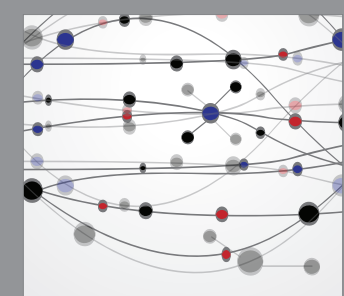

The Scientific World Journal
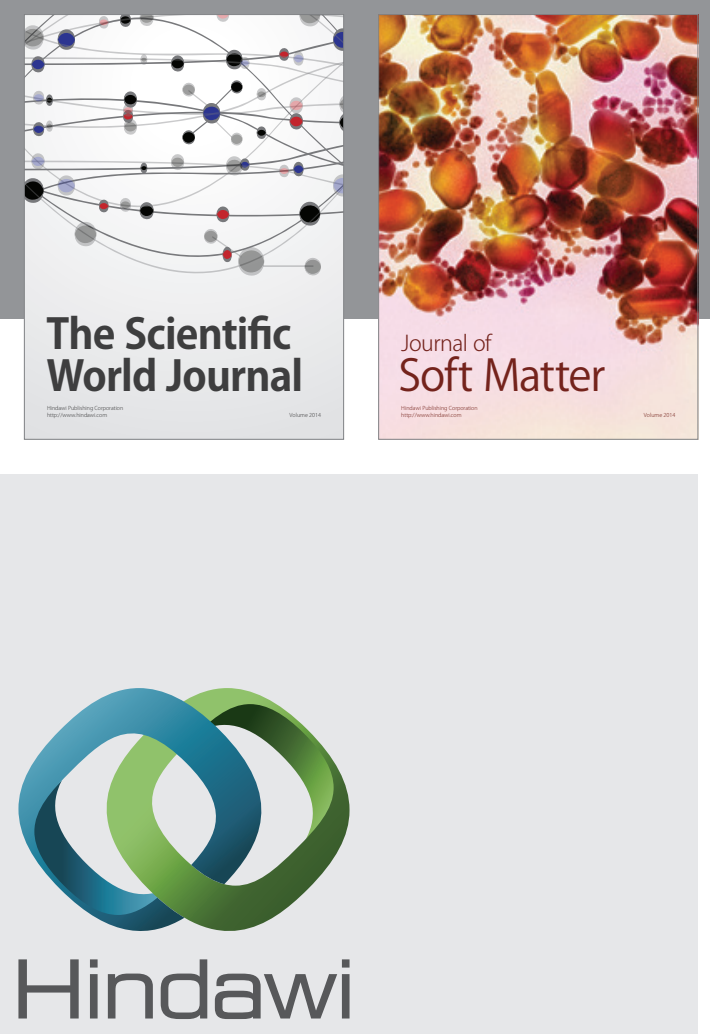

Submit your manuscripts at

http://www.hindawi.com
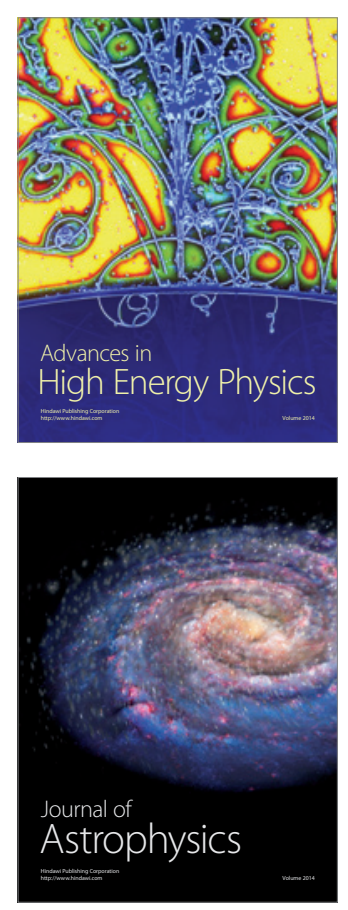
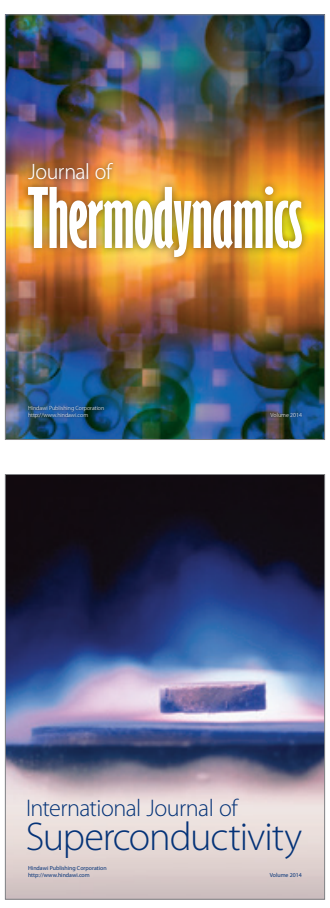
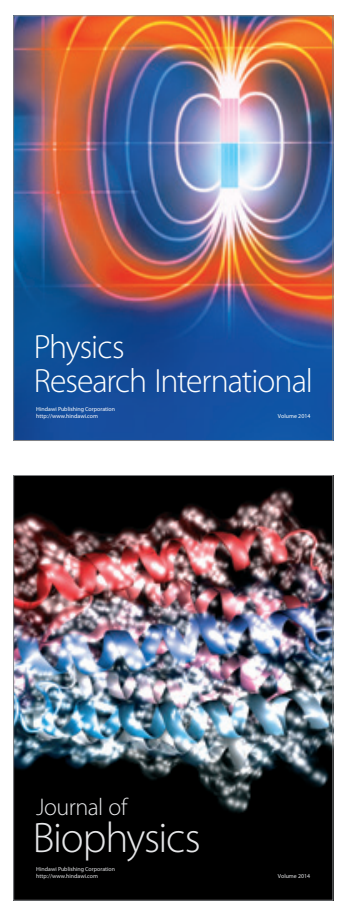
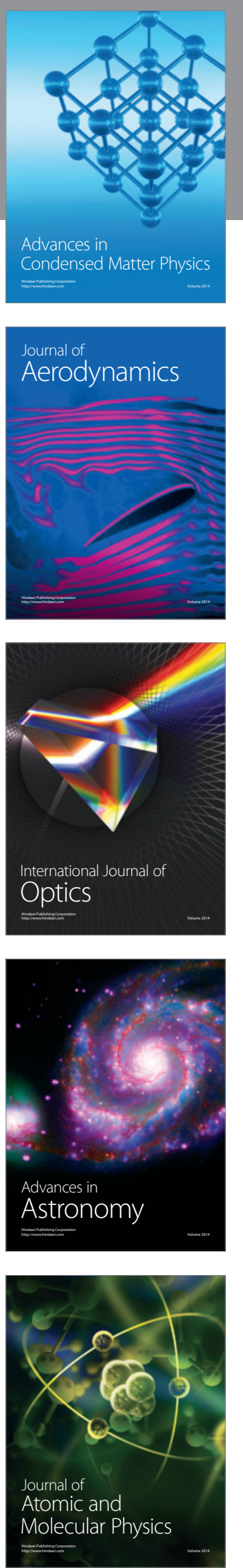